\title{
Performance Capture for Virtual Heritage: Virtual re-enactment for the lost film Pontianak
}

\author{
Benjamin Seide \\ Nanyang Technological University \\ Singapore \\ bseide@ntu.edu.sg
}

\author{
Benjamin Slater \\ Nanyang Technological University \\ Singapore \\ baslater@ntu.edu.sg
}

\begin{abstract}
Our project created an early $20^{\text {th }}$ century Malay-Singaporean Kampong village as a virtual environment. In this paper, we outline our approach of capturing performances to re-enact scenes for a virtual reality experience of the believed to be lost horror film Pontianak (1957).
\end{abstract}

Virtual reality. Cultural heritage. Immersive experience. Performance. Motion capture.

\section{INTRODUCTION}

Virtual heritage projects are successfully reconstructing and re-creating environments and objects of cultural significance. Technical advancements over the past decades in hard and software, from photogrammetry, laser scanning, drones, CG animation, VR headsets to GPUs and game engines have contributed to broader accessibility and overall increased visual fidelity of virtual heritage environments, leading to new experiences considered visually photorealistic (See i.e. photogrammetry projects of Raiz New Media). Inhabiting such photorealistic virtual environments with realistic-appearing actors will enable Virtual Heritage to attract a broader audience beyond researchers, creating accessible reconstructions for museum installations and edutainment.

Bogdanovych et al. (2010) argue that populating a Virtual Heritage site with virtual agents could bring heritage preservation to a new level but found that previous attempts were unsatisfying as they "are not involved into historically authentic interactions" and "that the agents are not behaving in a way that the relevant cultural knowledge is authentically presented to the human observer". The MIRALab mixed-reality project of Magnenat-Thalmann and Papagiannakis experiments with "animated characters acting a storytelling drama on the site of ancient Pompeii" and they conclude that "dramaturgical notions" could help to "develop an exciting edutainment medium" from their Virtual Heritage application. Researchers are aware that the audience's narrative involvement is relevant for a successful Virtual Heritage application.
In 2019, our research project began to investigate if approaches used in Virtual Heritage, such as the re-creation of virtual environments of historic sites, and developments in the field of game development, such as inhabiting virtual environments with actors and stories, could be combined and utilised to create Virtual Cinematic Heritage for films, which are considered lost and for which Film Heritage, in form of film preservation, is not an option. As a case study, the research begun the process of creating a Virtual Reality application for the historic Malayan Pontianak films produced in Singapore, originating in 1957 and originally starring Maria Menado as the titular supernatural creature from the oral folklore of Malaya. The visual transformation of the lead actress into the Pontianak creature amazed the audience in cinemas (Mustafar 2012).

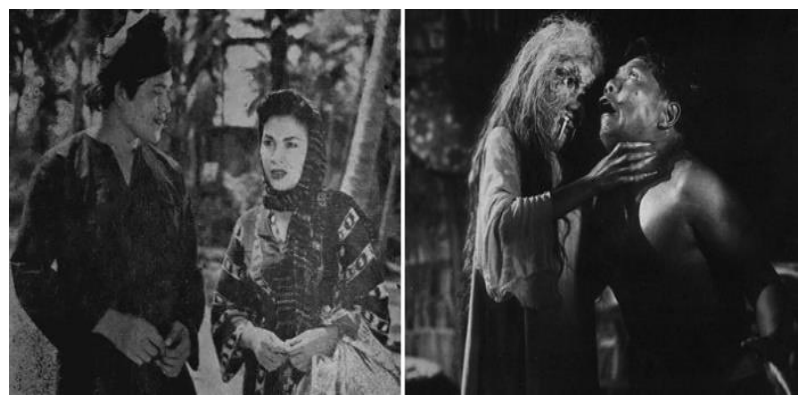

Figure 1: Film stills from Pontianak (1957) and Dendam Pontianak (1957) (C) 1957, Cathay-Keris

The first two films in this hugely popular trilogy Pontianak and Dendam Pontianak (Figure 1), both directed by B.N. Rao and starring Maria Menado, 
are believed to be "lost films" despite their relatively wide distribution in 1957 (Barnard, 2011). The choice of the Cathay-Keris Pontianak trilogy as a case study for the Virtual Cinematic Heritage application required the project to research the films' history, unearthing fragments of the lost synopses and based on those findings - to start the process of recreating scenes as a virtual representation with digital reincarnations of the actors and their performances.

Creating believable digital actors, although significant advancements have been made in recent years, is still considered a hugely challenging task. In 2018, Epic demonstrated with their Andy Serkis and Siren Digital Human projects, that convincing realism was achievable, most impressively in a real-time game engine environment. Epic Games went as far as to announce that "crossing the uncanny valley in realtime" is possible (Epic, 2018). While many factors contribute to the realism of a Computer-generated (CG) character, among them skin and eye textures and shaders, the hair and cloth simulation, the character's level of detail, Epic's Digital Human demonstrated once more that using the performance and facial expression of real actors would be one of the deciding factors of believability. At the same time, very affordable motion capture alternatives entered the market. These developments have huge implications for virtual heritage applications: are realistic digital humans within reach for non-commercial, artistic and smaller academic research teams?

In our previously published findings, we outlined steps and processes involved in creating the Virtual Cinematic Heritage Application of the Pontianak snake bite scene as a Virtual Reality experience (Seide, Slater 2020). Since these earlier tests, our project went through several iterations of capturing performances with actors and gained new knowledge. While our investigations are ongoing, in this paper, we will describe key considerations, issues and further findings, focusing on our investigation in performance capture and virtual reenactment of key scenes for the Virtual Cinematic Heritage Application.

\section{PERFORMANCE CAPTURE}

The two motion capture systems available to and evaluated by the project are a camera-based system from Vicon, which is a permanent setup in our research facilities, and as a second system, the portable inertial sensor-based system from Rokoko, which is considered an entry-level low-cost alternative to the more expensive professional camera-based system. Skogstad and Nymoen analyse both concepts and conclude "If high positional precision is required, OptiTrack (a camera-based system) is preferable over Xsens (a sensor-based system), but [...] Xsens provides less noisy data without occlusion problems" [xxx]. The two specific systems compared here by Skogstad and Nymoen, OptiTrack and Xsens, are indeed a fair comparison as both can be considered in a similar cost category; in contrast, our two systems from Vicon and Rokoko would not be considered as such. However, the lower cost Rokoko system is promoted as an alternative to the more expensive camera-based systems and the portability feature is an advantage that has to be considered, and as we aimed to capture actors walking within a large area, the portable sensor-based system appeared more practical for our use case.

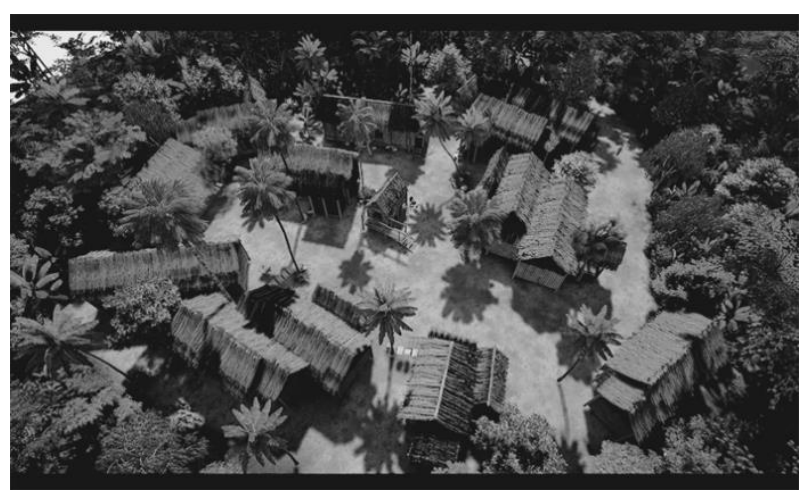

Figure 2: Virtual Kampong Village, 2020, Benjamin Seide, still image from VR experience, (C) 2020, the authors.

\section{Capturing the Pontianak Snake Bite scene}

Our goal was to capture the entire 3-minute-long scene with the two actors talking while walking through the virtual kampong village (Figure 2) in one continuous take. Following the serval test, the outdoor sports field on our university campus was chosen as capturing area as it provided the necessary open space, power outlets and with the grass field a relatively soft area for the actor to fall on. Electricity is only required for the WIFI router, which connects the suit with the recording laptop. During our tests, we did power the router with a portable UPS battery but decided that longer power cables would be a more reliable solution.

Capturing outdoor in a wide-open space had another significant advantage, Rokoko's sensorbased suits are highly sensitive to electromagnetic interference. Although the recommended distance to metallic objects is only 1 meter, we could not establish any entirely interference-free capturing area anywhere in our school's building. In our studio space, approx. 10 by 10 meters, equipped with the Vicon motion capture system, only a very small "sweet spot" area could be identified, in which the system would report an acceptable low amount 
of interference. This "sweet spot" area in our studio is so small that walking or dancing is not an option, the actor can only stand or sit without causing too much interference. However, we identified the school's auditorium stage as an area with an acceptable low amount of interference for performances that require more space.

As a final result in the virtual reality application, we planned for our actors to stroll through the virtual kampong village, and thus, to match the walking performance with the layout of the village, and to avoid the actors to collide with virtual buildings and trees, the walking path through the model of the village was translated into a top view map with a 2D coordinate system, and from there to the sports field, marking checkpoint and boundary and spanning an area of 40 by 25 meters for the actors to walk in.

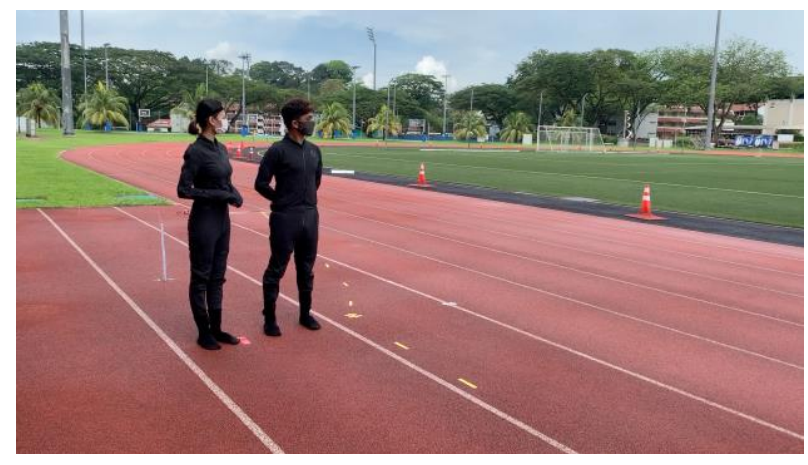

Figure 3: Outdoor Performance Capture, (C) 2020, the authors.

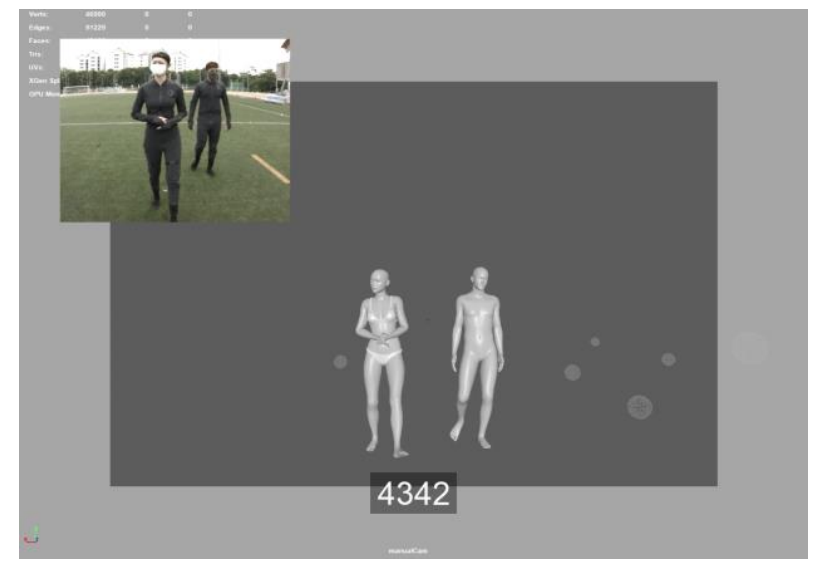

Figure 4: Performance Capture retargeted, (C) 2020, the authors.

A more sophisticated approach of matching an actor's position with the virtual environment during the capturing is to create a live preview by streaming the motion capture performance into a real-time virtual environment of i.e. unreal engine or unity. This live preview process is considered a type of "Virtual Production". The Rokoko system supports this forwarding of the capture data to Unreal Engine with its easy-to-use software and game engine plugin for the receiving side. However, sensor-based systems do not provide a reliable "absolute" position in "world" space. For our particular use case with two actors walking side-byside for minutes in a large area, the so-called "drifting" causes the 2 actors' captured position to be meters apart over time - while in reality, they are still just centimetres next to each other. Rokoko offers a smart solution to provide the "absolute" position by supporting SteamVR; HTC Vive trackers mounted on the actor, objects or a virtual camera can be utilised within the Rokoko software, as well as forwarded to the receiving game engine. As the SteamVR setup requires HTC base stations, it ultimately means the setup transforms into a combination of sensor and camera-based. For our particular application with the $40 \mathrm{~m} \times 25 \mathrm{~m}$ large volume, a SteamVR base station setup was not a serious consideration; apparently, HTC experimented back in 2018 with a 16 base station setup, but the officially supported HTC setup is still 4 base stations for $10 \mathrm{~m} \times 10 \mathrm{~m}$.

As a result of capturing without "absolute" position, the drift between the two characters accumulated to several meters over the entire capture time and thus required us to scale and reposition the data extensively to fit the village layout. Once these corrections were done, we prepared a video (Figure 4) of the captured characters for the voiceover acting and facial capture at the sound recording studio.

Our workflow at the time of recording was to capture the facial data transmitted from an iPhone to Reallusion iClone 7 (Figure 5), a decision supported by the fact that the base models of the 3D characters were created with Reallusion software and followed the blend shape model required for the facial capturing. The Rokoko system supports the body, facial and finger capturing simultaneously, and at the time of writing, we are experimenting with the full setup of all components: bodysuit, finger gloves and iPhone FacelD-based facial capture.

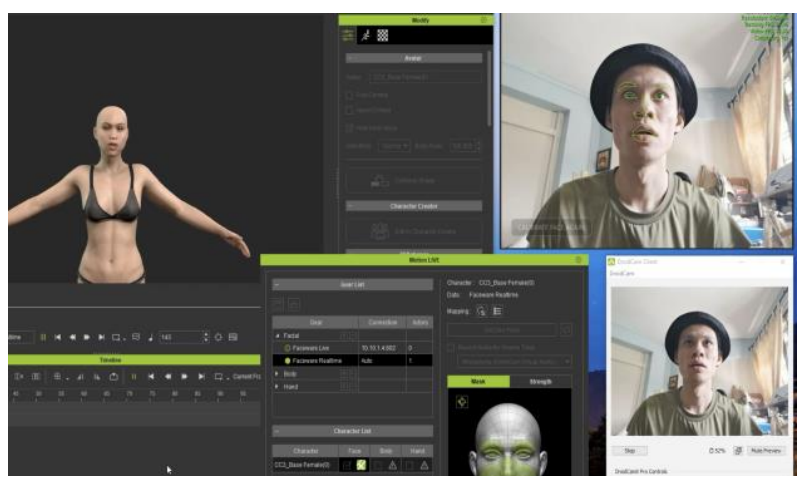

Figure 5: Facial Capture in Reallusion iClone, (C 2020, the authors. 


\section{REFERENCES}

Barnard, T. P. (2011) Films of change in early Singaporean film history, pp. 47-48. In Y. Michalik (ed.), Singapore independent film. Marburg [Germany]: Schüren.

Raiz New Media. Website:

http://www.raiznewmedia.com/najera.html (retrieved 10 March 2021).

Bogdanovych, A., Rodriguez-Aguilar, J. A., Simoff, S. and Cohen, A. (2010). Authentic Interactive Reenactment of Cultural Heritage with 3D Virtual Worlds and Artificial Intelligence, Applied Artificial Intelligence, 24 (6), pp. 617-647.

Epic Games Digital Humans project. Unreal Engine website: $\quad$ https://docs.unrealengine.com/enUS/Resources/Showcases/DigitalHumans/index.ht $\mathrm{ml}$ (retrieved 17 January 2020).

Magnenat-Thalmann, N., Papagiannakis, G. (2005) Virtual Worlds and Augmented Reality in Cultural Heritage Applications, pp. 2-9.
Mustafar, A.R. 50 TAHUN FILEM MALAYSIA \& SINGAPURA (1930-1980), Pekan IImu Publications, Malaysia: 2019, p. 114

Reallusion Face Mocap. Website: https://mocap.reallusion.com/iclone-motion-livemocap/iphone-live-face.html (retrieved 17 January 2020)

Seide B., Slater B. (2020) "Virtual Cinematic Heritage for the Lost Singaporean Film Pontianak (1957)". In Rauterberg M. (eds.) Culture and Computing. HCII 2020. Lecture Notes in Computer Science, 12215. Springer, Cham, pp. $396-414$.

Skogstad, S. \& Nymoen, K. \& Høvin, M. (2011) Comparing Inertial and Optical MoCap Technologies for Synthesis Control. Proceedings of the 8th Sound and Music Computing Conference, SMC (2011).

Unreal Engine (2018) News blog, April 2018. Website: https://www.unrealengine.com/enUS/events/siren-at-fmx-2018-crossing-theuncanny-valley-in-real-time (retrieved 17 January 2020). 